\title{
Simultaneous state and unknown inputs estimation with PI and PMI observers for Takagi Sugeno model with unmeasurable premise variables
}

\author{
Dalil Ichalal, Benoît Marx, José Ragot and Didier Maquin
}

\begin{abstract}
In this paper, a proportional integral (PI) and a proportional multiple integral observer (PMI) are proposed in order to estimate the state and the unknown inputs of nonlinear systems described by a Takagi-Sugeno model with unmeasurable premise variables. This work is an extension to nonlinear systems of the PI and PMI observers developed for linear systems. The state estimation error is written as a perturbed system. First, the convergence conditions of the state estimation errors between the system and each observer are given in LMI (Linear Matrix Inequality) formulation. Secondly, a comparison between the two observers is made through an academic example.
\end{abstract}

\section{INTRODUCTION}

Model-based approaches have been important and useful means to construct a fault diagnosis module for nonlinear systems in order to detect, isolate and identify actuator, sensor and system faults. Generally, the implementation of these functions is realized with observers. Moreover, observers provide an estimation of accessible and inaccessible states, outputs and faults of nonlinear systems. The estimated signals are used for example to elaborate feedback control laws, fault detection and isolation procedure (FDI) and fault tolerant control (FTC) [18], [10].

The proposed work focuses on the class of nonlinear systems described by Takagi-Sugeno models [22] with unmeasurable premise variables. The T-S model provides a useful tool to represent with a good precision a large class of nonlinear systems [23] and can even describe exactly certain classes of nonlinear systems [24] by using the nonlinear sector transformation. In the recent years, considerable efforts have been provided to study stability and stabilization of this class of systems [14], [23], [8], [5]. The topic of state estimation has also been widely studied in many works. In [2], [19], [16], [3], the authors proposed different methods in order to estimate the state of T-S systems for the purpose of diagnosis.

The main advantage of T-S structure is its simplicity because it originates from the interpolation between linear systems. Thus, analysis and design methods developed for linear systems can be generalized to nonlinear systems as used in the works cited above.

This research was supported by the TASSILI No. 07 program under MDU grant 714 .

The authors are with Centre de Recherche en Automatique de Nancy (CRAN), Nancy-Université, CNRS, 2 avenue de la forêt de Haye F-54516 Vandoeuvre-les-Nancy. \{dalil.ichalal, benoit.marx, jose.ragot, didier.maquin\}eensem. inpl-nancy. fr
In the context of robust observer design, one of the most successful technique is the use of PI observer, in which the unknown inputs are estimated simultaneously with the states of the system. The PI observer was first proposed by Wojciechowsky in [25] for single input-single output LTI systems. A generalization scheme was performed by Kaczorek [12] to multivariable systems. Thereafter, the PI observer has been used in different studies. In [20] a linear PI observer is designed and applied to a physical system. In [15] a PI observer for linear descriptor systems is proposed. However, this observer can be used only if the unknown inputs are constant over the time, nevertheless in practical cases the approach is effective if the variations of the unknown inputs are slow in respect to the dynamic of the system. In other cases, this problem can be solved by using multiple integrals in the observer in order to estimate all of the derivatives of the unknown inputs. A PMI observer was firstly proposed by Jiang in [11]. In [7], [13] a proportional multiple integral observer is proposed to estimate a large class of signals described in a polynomial form for LTI descriptor systems. An other paper [9], presented in the same conference, deals with the state estimation using a new method consisting in the transformation of the TS system with unmeasurable premise variables into an uncertain TS system with estimated premise variables, in addition, the method is extended to estimate the unknown input using a PI observer.

We propose, in this paper, a generalization of the PI and PMI observers to nonlinear systems described by T-S models with unmeasurable premise variables. The paper is organized as follows. Section II presents the T-S structure and the problem of state estimation, and gives the motivation of this work. In section III.A the design of PI observer is addressed and in section III.B the PMI observer is studied. Section IV presents a numerical example with discussion about the performances of the two proposed observers. Finally, this note is ending with conclusions and perspectives. The idea is based on two steps: the first step consists to transform the TS system with unmeasurable premise variable into a perturbed TS system with estimated premise variable. The perturbation term is due to the unmeasurable premise variable. The second step is to make the system in an augmented form by adding integrators to estimate the unknown input. 


\section{PRELIMINARIES AND PROBLEM STATEMENT}

\section{A. Multiple model approach}

Consider the following general form of continuous-time nonlinear systems:

$$
\left\{\begin{array}{l}
\dot{x}(t)=f(x(t), u(t)) \\
y(t)=h(x(t), u(t))
\end{array}\right.
$$

where $x \in \mathbb{R}^{n}, u \in \mathbb{R}^{m}, y \in \mathbb{R}^{q}$ and $f$ and $h$ are nonlinear functions. The representation (1) is difficult to study, elsewhere in literature, all of the works developed concerning the nonlinear systems concern specific classes. For example, in [1], [21], Lipschitz systems, which are represented by a linear part and a nonlinear one, are considered. The nonlinear part is assumed to be Lipschitz with respect to the state $x$.

As mentioned in the introduction, the T-S model approach is a very interesting method to represent nonlinear systems. Different methods exist to obtain a T-S model, as identification or linearization of the system (1) around different operating points or by using the nonlinear sector transformation. The multiple model structure is given by:

$$
\left\{\begin{array}{l}
\dot{x}(t)=\sum_{i=1}^{r} \mu_{i}(\xi(t))\left(A_{i} x(t)+B_{i} u(t)+E_{i} d(t)+W_{i} \omega(t)\right) \\
y(t)=C x(t)+D u(t)+G d(t)+W \omega(t)
\end{array}\right.
$$

where $A_{i} \in \mathbb{R}^{n \times n}, B_{i} \in \mathbb{R}^{n \times m}, C \in \mathbb{R}^{q \times n}, D \in \mathbb{R}^{q \times m}, E_{i} \in \mathbb{R}^{n \times s}$, $W_{i} \in \mathbb{R}^{n \times v}$ and $G \in \mathbb{R}^{q \times s}$, and $W \in \mathbb{R}^{q \times v}$. The unknown inputs are modeled by $d(t)$ and $\omega(t)$ are the noises affecting the state and the measurement equation. In this structure, the output is assumed to be linear with regard to the state of the system. The weighing functions $\mu_{i}$ are nonlinear and depend on the decision variable $\xi(t)$ which can be measurable like $\{u(t), y(t)\}$ or not measurable like the state $x(t)$ of the system. The weighting functions satisfy the following properties:

$$
\left\{\begin{array}{c}
0 \leq \mu_{i}(\xi(t)) \leq 1 \\
\sum_{i=1}^{r} \mu_{i}(\xi(t))=1
\end{array}\right.
$$

Thus the structure of the multiple model is simple and is considered as a universal approximator since it can represent any nonlinear behavior according to an adequate number $r$ of submodels. The multiple model structure provides a mean to generalize the tools developed for linear systems to nonlinear systems due to the properties expressed in (3).

\section{B. Problem statement}

Diagnosis of nonlinear systems is often based on a bank of observers to detect and isolate actuator and sensor faults. For designing observers, it is often assumed, in the literature that the weighting functions $\mu_{i}$ depend on measurable premise variables $u$ and/or $y$. Thus, to perform diagnosis, it is necessary to develop two different multiple models. The first one where the weighting functions depend only on the output of the system in order to detect and isolate actuator faults. The second one with weighting functions depending only on the input of the system in order to detect and isolate sensor faults. To reduce this difficulty, it is interesting to develop only one multiple model using weighing functions which depend on the state of the system. Thus, the same multiple model can be used to construct observer bank for detecting and isolating both actuator and sensor faults. However, the main difficulty is due to the fact that the state equation is now a nonlinear function of the state. In the literature, only few works are developed for observer design for T-S systems with unmeasurable premise variables. Nevertheless, we can cite [16], [17], [26], [4], where the authors re-write the system either as a perturbed or uncertain T-S system with measurable premise variables.

\section{MAIN RESULT}

Along this paper, we assume that the following assumptions hold:

- A1. The system is stable

- A2. The signals $u(t), d(t)$ and $\omega(t)$ are bounded.

Practically, these assumptions are often not restrictive.

\section{A. Extension of classical PI observer}

Consider the following T-S fuzzy system with weighting functions $\mu_{i}$ depending on the state of the system:

$$
\left\{\begin{aligned}
\dot{x}(t) & =\sum_{i=1}^{r} \mu_{i}(x(t))\left(A_{i} x(t)+B_{i} u(t)+E_{i} d(t)+W_{i} \omega(t)\right) \\
y(t) & =C x(t)+G d(t)+W \omega(t)
\end{aligned}\right.
$$

In the next, for sake of simplicity, the time variable $t$ is omitted.

In this section, the unknown inputs $d(t)$ are assumed to be constant:

- A3. $\dot{d}=0$

This assumption will be relaxed in the section III.B.

The proposed PI observer is given by the following equations:

$$
\left\{\begin{array}{l}
\dot{\hat{x}}=\sum_{i=1}^{r} \mu_{i}(\hat{x})\left(A_{i} \hat{x}+B_{i} u+E_{i} \hat{d}+K_{P i}(y-\hat{y})\right) \\
\hat{y}=C \hat{x}+G \hat{d} \\
\dot{\hat{d}}=\sum_{i=1}^{r} \mu_{i}(\hat{x}) K_{I i}(y-\hat{y})
\end{array}\right.
$$

where $\hat{x}$ and $\hat{d}$ are the estimates of $x$ and $d$. In order to facilitate the comparison between the system and its observer, the system (4) can be written as a perturbed system with weighting functions $\mu_{i}$ depending on the estimated state as follows:

$$
\dot{x}=\sum_{i=1}^{r} \mu_{i}(\hat{x})\left(A_{i} x+B_{i} u+E_{i} d+W_{i} \omega+v\right)
$$

where:

$$
v=\sum_{i=1}^{r}\left(\mu_{i}(x)-\mu_{i}(\hat{x})\right)\left(A_{i} x+B_{i} u+E_{i} d+W_{i} \omega\right)
$$

This term is seen as a bounded vanishing perturbation to minimize. Indeed, due to the assumptions A1, A2 and the definition of the weighting functions (3), $v(t)$ is bounded and if $\hat{x} \rightarrow x$ then $v \rightarrow 0$. 
The assumption A3 allows to make the system (6) in the augmented form:

$$
\left\{\begin{array}{l}
\dot{x}_{a}=\sum_{i=1}^{r} \mu_{i}(\hat{x})\left(\tilde{A}_{i} x_{a}+\tilde{B}_{i} u+\tilde{\Gamma}_{i} \tilde{\omega}\right) \\
y=\tilde{C} x_{a}+\tilde{D} \tilde{\omega}
\end{array}\right.
$$

where:

$$
\begin{gathered}
\tilde{A}_{i}=\left[\begin{array}{cc}
A_{i} & E_{i} \\
0 & 0
\end{array}\right], \tilde{B}_{i}=\left[\begin{array}{c}
B_{i} \\
0
\end{array}\right], \tilde{\Gamma}_{i}=\left[\begin{array}{cc}
I & W_{i} \\
0 & 0
\end{array}\right], \tilde{\omega}=\left[\begin{array}{c}
v \\
\omega
\end{array}\right] \\
\tilde{C}=\left[\begin{array}{ll}
C & G
\end{array}\right], \tilde{D}=\left[\begin{array}{ll}
0 & W
\end{array}\right], x_{a}=\left[\begin{array}{c}
x \\
d
\end{array}\right]
\end{gathered}
$$

A similar reasoning makes it possible to transform the proposed PI observer (5) in the following augmented form:

$$
\left\{\begin{array}{l}
\dot{\hat{x}}_{a}=\sum_{i=1}^{r} \mu_{i}(\hat{x})\left(\tilde{A}_{i} \hat{x}_{a}+\tilde{B}_{i} u+\tilde{K}_{i}(y-\hat{y})\right) \\
\hat{y}=\tilde{C} \hat{x}_{a}
\end{array}\right.
$$

where:

$$
\tilde{K}_{i}=\left[\begin{array}{c}
K_{P i} \\
K_{I i}
\end{array}\right]
$$

Let us consider the augmented state estimation error:

$$
e_{a}=x_{a}-\hat{x}_{a}
$$

whose dynamics is given by:

$$
\dot{e}_{a}=\sum_{i=1}^{r} \mu_{i}(\hat{x})\left(\left(\tilde{A}_{i}-\tilde{K}_{i} \tilde{C}\right) e_{a}+\left(\tilde{\Gamma}_{i}-\tilde{K}_{i} \tilde{D}\right) \tilde{\omega}\right)
$$

The goal is to determine the gain matrices $\tilde{K}_{i}$ of the observer in order to stabilize the system (11), i.e. to guarantee the convergence of the state estimation error toward zero when the perturbation $\tilde{\omega}$ is nul and to attenuate the transfer gain from the bounded perturbation $\widetilde{\omega}(t)$ to the state estimation error $e_{a}(t)$ when $\tilde{\omega}(t)$ is different from zero $(\tilde{\omega}(t)$ is bounded since assumptions $\mathbf{A 1}$ and $\mathbf{A 2}$ are satisfied).

In order to establish the existence conditions of the PI observer in theorem 1 , let us first introduce the following lemma:

Lemma 1: [24] Consider the continuous-time TS-system defined by:

$$
\left\{\begin{aligned}
\dot{x}(t) & =\sum_{i=1}^{r} \mu_{i}(x(t))\left(A_{i} x(t)+B_{i} u(t)\right) \\
y(t) & =C x(t)
\end{aligned}\right.
$$

The system (12) is stable and verifies the $\mathscr{L}_{2}$-gain condition: $\|y(t)\|_{2}<\gamma\|u(t)\|_{2}$ if there exists a symmetric positive definite matrix $P$ such that (13) is satisfied for $i=1, \ldots, r$ :

$$
\left[\begin{array}{cc}
A_{i}^{T} P+P A_{i}+C^{T} C & P B_{i} \\
B_{i}^{T} P & -\gamma^{2} I
\end{array}\right]<0
$$

Theorem 1: The PI observer (9) for the system (8) is determined by minimizing $\bar{\gamma}$ under the following LMI constraints in the variables $P=P^{T}>0, M_{i}$ and $\bar{\gamma}$ for $i=1, \ldots, r$ :

$$
\left[\begin{array}{cc}
\tilde{A}_{i}^{T} P+P \tilde{A}_{i}-M_{i} \tilde{C}-\tilde{C}^{T} M_{i}+I & P \tilde{\Gamma}_{i}-M_{i} \tilde{D} \\
\tilde{\Gamma}_{i}^{T} P-\tilde{D}^{T} M_{i}^{T} & -\bar{\gamma} I
\end{array}\right]<0
$$

The gains of the observer are derived from $\tilde{K}_{i}=P^{-1} M_{i}$ and the attenuation level is calculated by $\gamma=\sqrt{\bar{\gamma}}$.

Proof: According to the assumptions A1 and A2, $\tilde{\omega}(t)$ is bounded. Then, by applying lemma 1 with $\left\|e_{a}(t)\right\|_{2}<$ $\gamma \|\left.\tilde{\omega}(t)\right|_{2}$, we obtain:

$$
\left[\begin{array}{cc}
\tilde{A}_{i}^{T} P+P \tilde{A}_{i}-P \tilde{K}_{i} \tilde{C}-\tilde{C}^{T} \tilde{K}_{i}^{T} P+I & P \tilde{\Gamma}_{i}-P \tilde{K}_{i} \tilde{D} \\
\tilde{\Gamma}_{i}^{T} P-\tilde{D}^{T} \tilde{K}_{i}^{T} P & -\gamma^{2} I
\end{array}\right]<0
$$

The LMI formulation in theorem 1 is obtained by using the following changes of variables:

$$
M_{i}=P \tilde{K}_{i}, \quad \bar{\gamma}=\gamma^{2}
$$

Remark 1: The minimization of $\gamma$ may result in slow dynamics of the state estimation error. This problem can be solved by pole assignment of the matrices $\left(\tilde{A}_{i}-\tilde{K}_{i} \tilde{C}\right)$ in the left half complex plane defined by:

$$
\{z \mid \operatorname{Re}(z)<-\lambda\}, \quad \lambda>0
$$

Thus, the LMIs in theorem 1 are solved simultaneously with the following constraint (to impose $\operatorname{Re}\left(\lambda_{i}\right)<-\lambda$, where $\lambda_{i}$ are the eigenvalues of $\tilde{A}_{i}$ and $\left.\lambda>0\right)$ :

$$
P\left(\tilde{A}_{i}+\lambda I\right)+\left(\tilde{A}_{i}+\lambda I\right)^{T} P-M_{i} \tilde{C}-\tilde{C}^{T} M_{i}^{T}<0
$$

More precise pole clustering can be obtained by adding LMI constraints [6].

This approach remains effective in practical cases where the assumption 1 is not satisfied. However, the unknown inputs must vary slowly. Otherwise, bad state and unknown inputs estimation are obtained by using this method. In the next section, another method to estimate the state and the unknown inputs is proposed. It is based on the proportional multiple integral observer. This observer is interesting because the assumption $\mathbf{A 3}$ is not required in the theoretic proof, so it is possible to estimate a larger class of unknown inputs.

\section{B. Proportional multiple integral observer}

Let us consider the multiple model with unmeasurable premise variables described in (4). The unknown input is assumed to be a bounded time varying signal with null $q^{\text {th }}$ derivative:

- A4. $d^{(q)}(t)=0$ 
Generally, the use of a PI observer requires the condition that the unknown input is constant (i.e.: $\dot{d}=0$ ), thus, the unknown inputs which satisfies $\mathbf{A 4}$ cannot be estimated with a good precision. Then, PMI observer is more adequate for this problem, because the observer estimates the $(q-1)^{t h}$ derivatives of the unknown input and gives a good precision of the estimated unknown inputs.

Consider the generalization of the proportional multipleintegral observer to T-S systems of the PMI observer proposed in [7] for linear descriptor systems:

$$
\left\{\begin{array}{l}
\dot{\hat{x}}=\sum_{i=1}^{r} \mu_{i}(\hat{x})\left(A_{i} \hat{x}+B_{i} u+E_{i} \hat{d}_{0}+K_{P i}(y-\hat{y})\right) \\
\hat{y}=C \hat{x}+G \hat{d} \\
\dot{\hat{d}_{0}}=\sum_{i=1}^{r} \mu_{i}(\hat{x}) K_{I i}^{0}(y-\hat{y})+\hat{d}_{1} \\
\dot{\hat{d}_{1}}=\sum_{i=1}^{r} \mu_{i}(\hat{x}) K_{I i}^{1}(y-\hat{y})+\hat{d}_{2} \\
\vdots \\
\dot{\hat{d}}_{q-2}=\sum_{i=1}^{r} \mu_{i}(\hat{x}) K_{I i}^{q-2}(y-\hat{y})+\hat{d}_{q-1} \\
\dot{\hat{d}}_{q-1}=\sum_{i=1}^{r} \mu_{i}(\hat{x}) K_{I i}^{q-1}(y-\hat{y})
\end{array}\right.
$$

where $\hat{d}_{i}, i=1,2, \ldots,(q-1)$ are the estimation of the $(q-1)$ first derivatives of the unknown input $d(t)$.

The state and unknown inputs estimation errors are:

$$
e=x-\hat{x}, e_{0}=\dot{d}-\dot{\hat{d}}_{0}, \ldots, e_{q-1}=\dot{d}_{q-1}-\dot{\hat{d}}_{q-1}
$$

Their dynamics are given in the following form:

$$
\left\{\begin{array}{l}
\dot{e}=\sum_{i=1}^{r} \mu_{i}(\hat{x})\left(\left(A_{i}-K_{P i} C\right) e+\left(\Gamma_{i}-K_{P i} \bar{W}\right) \tilde{\omega}+\left(E_{i}-K_{P i} G\right) e_{0}\right) \\
\dot{e}_{0}=\sum_{i=1}^{r} \mu_{i}(\hat{x})\left(-K_{I i}^{0} C e+e_{1}-K_{I i}^{0} \bar{W} \tilde{\omega}-K_{I i}^{0} G e_{0}\right) \\
\dot{e}_{1}=\sum_{i=1}^{r} \mu_{i}(\hat{x})\left(-K_{I i}^{1} C e+e_{2}-K_{I i}^{1} \bar{W} \tilde{\omega}-K_{I i}^{1} G e_{0}\right) \\
\vdots \\
\dot{e}_{q-2}=\sum_{i=1}^{r} \mu_{i}(\hat{x})\left(-K_{I i}^{0} C e+e_{q-1}-K_{I i}^{q-2} \bar{W} \tilde{\omega}-K_{I i}^{q-2} G e_{0}\right) \\
\dot{e}_{q-1}=\sum_{i=1}^{r} \mu_{i}(\hat{x})\left(-K_{I i}^{q-1} C e-K_{I i}^{0} \bar{W} \tilde{\omega}-K_{I i}^{q-1} G e_{0}\right)
\end{array}\right.
$$

where:

$$
\Gamma_{i}=\left[\begin{array}{ll}
I_{n} & W_{i}
\end{array}\right], \bar{W}=\left[\begin{array}{ll}
0 & W
\end{array}\right]
$$

The equations (19) can be rewritten in the following augmented form:

$$
\begin{aligned}
\dot{\tilde{e}} & =\sum_{i=1}^{r} \mu_{i}(\hat{x})\left(\left(\tilde{A}_{i}-\tilde{K}_{i} \tilde{C}\right) \tilde{e}+\left(\tilde{\Gamma}_{i}-\tilde{K}_{i} \bar{W}\right) \tilde{\omega}\right) \\
{\left[\begin{array}{c}
e \\
e_{0}
\end{array}\right] } & =\bar{C} \tilde{e}
\end{aligned}
$$

where:

$$
\begin{gathered}
\tilde{e}=\left[\begin{array}{c}
e \\
e_{0} \\
e_{1} \\
\vdots \\
e_{q-2} \\
e_{q-1}
\end{array}\right], \tilde{A}_{i}=\left[\begin{array}{cccccc}
A_{i} & E_{i} & 0 & \cdots & 0 & 0 \\
0 & 0 & I_{s} & \cdots & 0 & 0 \\
0 & 0 & 0 & \ddots & 0 & 0 \\
\vdots & \vdots & \vdots & \vdots & \vdots & \vdots \\
0 & 0 & 0 & 0 & 0 & I_{s} \\
0 & 0 & 0 & 0 & 0 & 0
\end{array}\right], \tilde{K}_{i}=\left[\begin{array}{c}
K_{P i} \\
K_{I i}^{0} \\
K_{I i}^{1} \\
\vdots \\
K_{I i}^{q-2} \\
K_{I i}^{q-1}
\end{array}\right] \\
\tilde{C}=\left[\begin{array}{cccccc}
C & G & 0 & \ldots & 0 & 0
\end{array}\right] \\
\tilde{\Gamma}_{i}=\left[\begin{array}{llll}
\Gamma_{i}^{T} & 0 & \ldots & 0
\end{array}\right]^{T}
\end{gathered}
$$

In the following, we are only interested with particular component $e$ and $e_{0}$ of $\tilde{e}$ :

$$
\left[\begin{array}{c}
e \\
e_{0}
\end{array}\right]=\bar{C} \tilde{e}
$$

where:

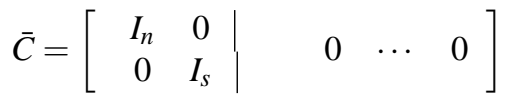

0 represents null matrix with appropriate dimensions.

Theorem 2: The PMI observer (18) for the system (8) that minimizes the transfer from $\tilde{\omega}(t)$ to $\left[e(t)^{T} \quad e_{0}(t)^{T}\right]$ is obtained by finding the matrices $P=P^{T}>0, M_{i}$ and $\bar{\gamma}$ that minimize $\bar{\gamma}$ under the following LMI constraints for $i=1, \ldots, r$ :

$$
\left[\begin{array}{cc}
\tilde{A}_{i}^{T} P+P \tilde{A}_{i}-M_{i} \tilde{C}-\tilde{C}^{T} M_{i}^{T}+\bar{C}^{T} \bar{C} & P \tilde{\Gamma}_{i}-M_{i} \bar{W} \\
\tilde{\Gamma}_{i}^{T} P-\bar{W}^{T} M_{i}^{T} & -\bar{\gamma} I
\end{array}\right]<0
$$

The gains of the observer are derived from:

$$
\tilde{K}_{i}=P^{-1} M_{i}
$$

and the attenuation level is calculated by:

$$
\gamma=\sqrt{\bar{\gamma}}
$$

Proof: The proof of theorem 2 is similar to the proof of theorem 1 by using the lemma 1 with the system (20)

Remark 2: When the condition $\mathbf{A 3}$ is not satisfied i.e. $d^{(q)} \neq 0$ but $d^{(q)}$ is bounded then, we can consider the $q^{\text {th }}$ derivative of $d(t)$ as a perturbation. The new perturbation vector is then given by:

$$
\tilde{\omega}(t)=\left[\begin{array}{lll}
v(t)^{T} & \omega(t)^{T} & d^{(q)}(t)^{T}
\end{array}\right]^{T}
$$

The additional component $d_{q}$ is added in the state vector. The matrices $\tilde{A}_{i}, \tilde{\Gamma}_{i}, \bar{W}, \tilde{C}$ are augmented. Then, the Theorem 2 can be applied in order to design the Proportional Multiple Integral Observer with minimization of the new bounded perturbation $\tilde{\omega}(t)$. 
IV. NUMERICAL EXAMPLE AND SIMULATIONS

In this section, the proposed method is illustrated through an academic example. Consider a continuous-time T-S system (4) defined by:

$$
\begin{gathered}
A_{1}=\left[\begin{array}{ccc}
-2 & 1 & 1 \\
1 & -3 & 0 \\
2 & 1 & -8
\end{array}\right], A_{2}=\left[\begin{array}{ccc}
-3 & 2 & -2 \\
5 & -3 & 0 \\
1 & 2 & -4
\end{array}\right], \\
B_{1}=\left[\begin{array}{c}
1 \\
5 \\
0.5
\end{array}\right], \quad B_{2}=\left[\begin{array}{c}
3 \\
1 \\
-7
\end{array}\right], E_{1}=\left[\begin{array}{ll}
0 & 7 \\
0 & 5 \\
0 & 2
\end{array}\right], \\
E_{2}=\left[\begin{array}{ll}
0 & 6 \\
0 & 3 \\
0 & 1
\end{array}\right], \quad W_{1}=W_{2}=\left[\begin{array}{l}
1 \\
1 \\
1
\end{array}\right], W=\left[\begin{array}{l}
0.5 \\
0.5
\end{array}\right],
\end{gathered}
$$

and

$$
C=\left[\begin{array}{lll}
1 & 1 & 1 \\
1 & 0 & 1
\end{array}\right], \quad G=\left[\begin{array}{ll}
5 & 0 \\
1 & 0
\end{array}\right]
$$

The unknown input vector $d(t)$ is made up of $d_{1}(t)$ which affects the outputs of the system and $d_{2}(t)$ affecting the dynamics of the system (see the matrices $E_{1}, E_{2}$ and $G$ ). For example, we can consider $d_{1}$ as a sensor fault and $d_{2}$ as an actuator one.

The weighting functions depend on the first component $x_{1}$ of the state vector $x$ and are defined as follows:

$$
\left\{\begin{array}{l}
\mu_{1}(x)=\frac{1-\tanh \left(x_{1}\right)}{2} \\
\mu_{2}(x)=1-\mu_{1}(x)
\end{array}\right.
$$

The weighting functions obtained without perturbations and unknown inputs are shown in figure 1. This figure shows that the system is clearly nonlinear since $\mu_{1}$ and $\mu_{2}$ are not constant functions.

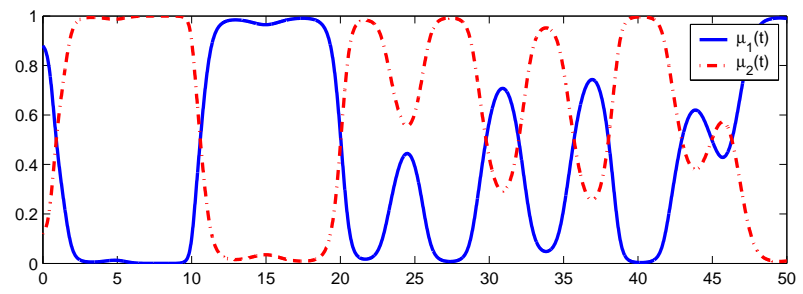

Fig. 1. Weighting functions $\mu_{1}$ and $\mu_{2}$

The perturbation $\omega$ is chosen as random signal uniformly distributed in $\left[\begin{array}{ll}-0.5 & 0.5\end{array}\right]$. The considered unknown inputs $d_{1}(t)$ and $d_{2}(t)$ are time varying signals with neglected fourth derivatives. After synthesizing a PI observer according to the theorem 1 and a PMI observer with $q=4$ according to the theorem 2, we obtain the simulation results depicted in the figures 2, 3, 4 and 5 .

Figures 2 and 3 show the unknown input and their estimations with PI and PMI observers. It is known that the
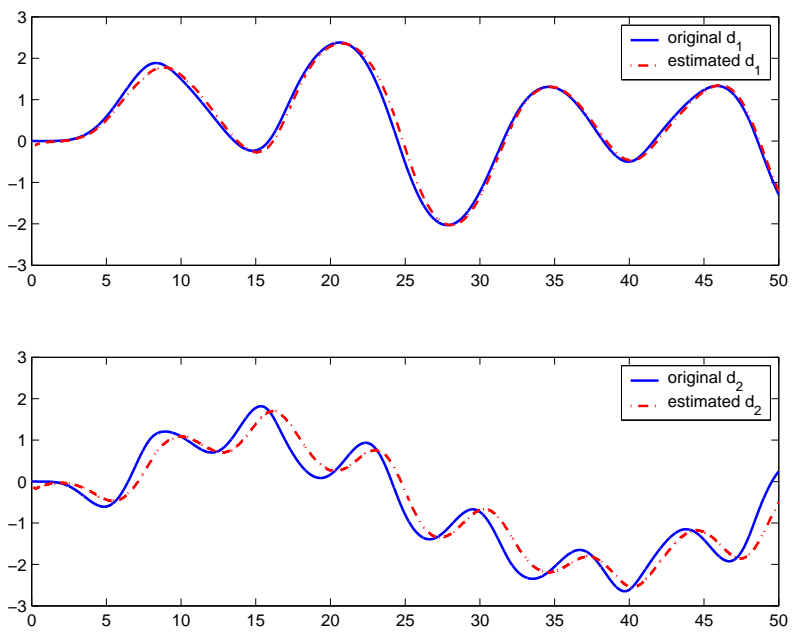

Fig. 2. Unknown input estimation with a PI observer
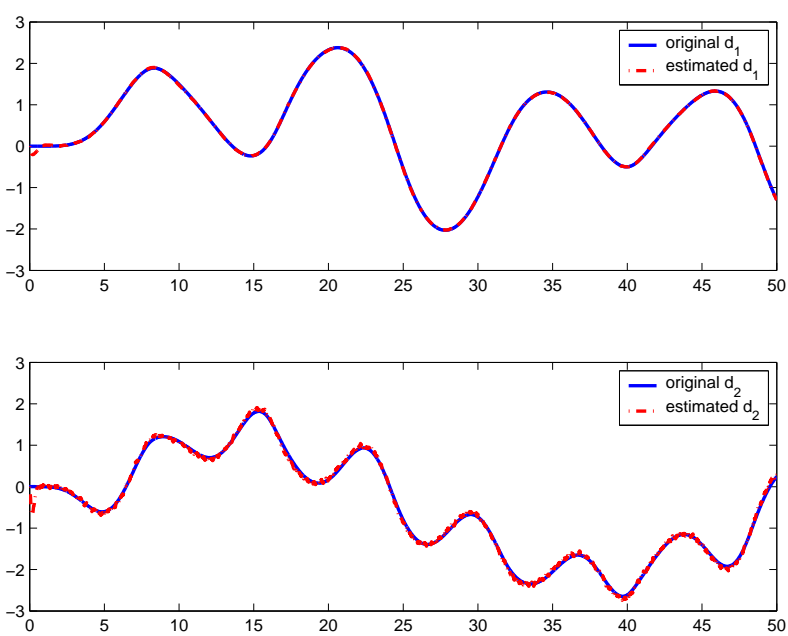

Fig. 3. Unknown input estimation with a PMI observer

PI observer gives an acceptable state and unknown inputs estimation even if the assumption A3 is not satisfied. However, in this example, the unknown inputs have fast variations resulting on bad state and unknown inputs estimation (figures 2 and 4) compared to the results given by the PMI observer (figures 3 and 5).

\section{CONCLUSIONS AND FUTURE WORKS}

The design of proportional integral (PI) and proportional multiple integrals (PMI) are studied in this paper. This work is an extension of the PI and PMI observers developed for linear systems to nonlinear T-S systems with unmeasurable premise variables. The convergence conditions of the state 


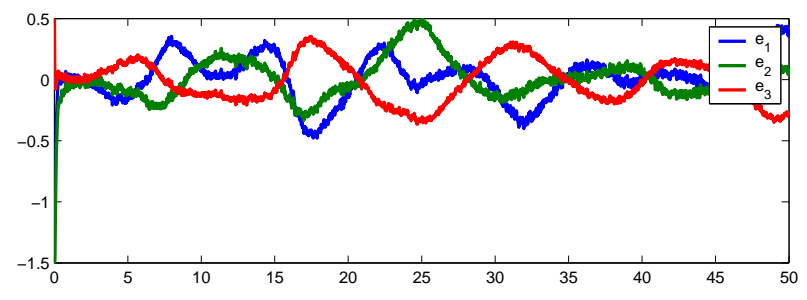

Fig. 4. State estimation error with a PI observer

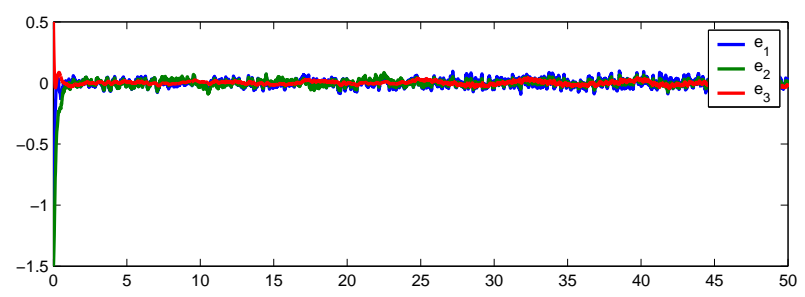

Fig. 5. State estimation error with a PMI observer

estimation error are given in the LMI formulation. The observers are robust since they are synthesized in order to minimize the effect of noises on the state estimation error by using an $\mathscr{L}_{2}$ approach. The PI observer is interesting for the estimation of constant or slowly varying unknown inputs and it is less sensitive to noises compared to the PMI observer [7]. In the other hand, PMI observer is a good way to obtain a more precise estimation of states and unknown inputs. The future works will concern, firstly, the improvement of the PMI observer by introducing a stable frequency weighting functions on the perturbations $\tilde{\omega}(t)$ which allows to reflect the expected frequency content of $\tilde{\omega}(t)$, secondly, the use of these observers in nonlinear system diagnosis.

\section{REFERENCES}

[1] M. Abbaszadeh and H. Marquez, "Robust $H_{\infty}$ observer design for a class of nonlinear uncertain systems via convex optimization," in American Control Conference, ACC'07, 2007.

[2] A. Akhenak, M. Chadli, J. Ragot, and D. Maquin, "Design of sliding mode unknown input observer for uncertain Takagi-Sugeno model," in 15th Mediterranean Conference on Control and Automation, MED'07, Athens, Greece, 2007.

[3] P. Bergsten, R. Palm, and D. Driankov, "Fuzzy observers," in IEEE International Fuzzy Systems Conference, Melbourne Australia, 2001.

[4] — , "Observers for Takagi-Sugeno fuzzy systems," IEEE Transactions on Systems, Man, and Cybernetics - Part B: Cybernetics, vol. 32, no. 1 , pp. 114-121, 2002.

[5] M. Chadli, D. Maquin, and J. Ragot, "Non quadratic stability analysis of Takagi-Sugeno systems," in IEEE Conference on Decision and Control, CDC'2002, Las Vegas, Nevada, USA, 2002.

[6] M. Chilali and P. Gahinet, "H-infinity design with pole placement constraints : an LMI approach," IEEE Transactions on Automatic Control, vol. 41, no. 3, pp. 358-367, 1996.
[7] Z. Gao and D. Ho, "Proportional multiple-integral observer design for descriptor systems with measurement output disturbances," IEE proceeding Control theory and application, vol. 151, no. 3, pp. 279288, 2004.

[8] T. Guerra, A. Kruszewski, L. Vermeiren, and H. Tirmant, "Conditions of output stabilization for nonlinear models in the Takagi-Sugeno's form," Fuzzy Sets and Systems, vol. 157, no. 9, pp. 1248-1259, May 2006.

[9] D. Ichalal, B. Marx, J. Ragot, and D. Maquin, "State and unknown input estimation for nonlinear systems described by Takagi-Sugeno models with unmeasurable premise variables." in 17th Mediterranean Conference on Control and Automation, MED'09, Thessaloniki, Greece, June 24-26 2009.

[10] R. Isermann, Fault-diagnosis systems: An introduction from fault detection to fault tolerance, Springer, Ed., 2007.

[11] G. Jiang, S. Wang, and W. Song, "Design of observer with integrators for linear systems with unknown input disturbances," Electronics Letters, vol. 36, no. 13, pp. 1168-1169, 2000.

[12] T. Kaczorek, "Proportional-integral observers for linear multivariable time-varying systems," Regelungstechnik, vol. 27, pp. 359-362, 1979.

[13] D. Koenig, "Unknown input proportional multiple-integral observer design for linear descriptor systems: application to state and fault estimation," IEEE Transactions on Automatic Control, vol. 50, no. 2, pp. 212-217, 2005.

[14] A. Kruszewski, "Lois de commande pour une classe de modèles non linéaires sous la forme Takagi-Sugeno : Mise sous forme LMI," Ph.D. dissertation, Université de Valenciennes et du Hainaut-Cambresis, 2006, (In french).

[15] B. Marx, D. Koenig, and D. Georges, "Robust fault diagnosis for linear descriptor systems using proportional integral observers," in 42nd IEEE Conference on Decision and Control, 2003.

[16] R. Palm and P. Bergsten, "Sliding mode observers for Takagi-Sugeno fuzzy systems." 9th IEEE International Conference on Fuzzy Systems, FUZZ IEEE 2000, San Antonio, TX, USA, 2000.

[17] R. Palm and D. Driankov, "Towards a systematic analysis of fuzzy observers," in 18th NAFIPS Conference, New York, NY, USA, 1999.

[18] R. Patton, P. Frank, and R. Clark, Fault diagnosis in dynamic systems: Theory and application. Prentice Hall international, 1989.

[19] R. Patton, J. Chen, and C. Lopez-Toribio, "Fuzzy observers for nonlinear dynamic systems fault diagnosis," in 37th IEEE Conference on Decision and Control, Tampa, Florida USA, 1998.

[20] G. Pechoto de Melo and T. Souza Morais, "Fault detection using state observers with unknown input, identified by orthogonal functions and PI observers," Brazilian Society of mechanical sciences and Engineering, 2007.

[21] R. Rajamani, "Observers for Lipschitz nonlinear systems," IEEE Transactions on Automatic Control, vol. 43, pp. 397-401, March 1998.

[22] T. Takagi and M. Sugeno, "Fuzzy identification of systems and its applications to modeling and control," IEEE Transactions on Systems, Man, and Cybernetics, vol. 15, pp. 116-132, 1985.

[23] K. Tanaka, T. Ikeda, and H. Wang, "Fuzzy regulators and fuzzy observers: Relaxed stability conditions and LMI-based designs," IEEE Transactions on Fuzzy Systems, vol. 6, no. 2, pp. 250-265, 1998.

[24] K. Tanaka and H. Wang, Fuzzy Control Systems Design and Analysis: A Linear Matrix Inequality Approach, J. Wiley and Sons, Eds., 2001.

[25] B. Wojciechowski, "Analysis and synthesis of proportional-integral observers for single-input-single-output time-invariant continuous systems," Ph.D. dissertation, Gliwice, Poland, 1978.

[26] J. Yoneyama, " $H_{\infty}$ output feedback control for fuzzy systems with immeasurable premise variables: Discrete-time case," Applied Soft Computing, vol. 8, no. 2, pp. 949-958, Mar. 2008. 